\title{
A combined radial collimator and cooled beryllium filter for neutron scattering
}

\author{
Felix Groitla,b, Emmanouela Rantsiouc ${ }^{\mathrm{c}}$, Marek Bartkowiak ${ }^{\mathrm{c}}$, Uwe Filges ${ }^{\mathrm{c}}$, \\ Dieter Graf ${ }^{c}$, Christof Niedermayer ${ }^{b}$, Christian Rüegg ${ }^{b, d}$, Henrik M. \\ Rønnow ${ }^{\text {a,e }}$ \\ ${ }^{a}$ Laboratory for Quantum Magnetism, École Polytechnique Fédérale de Lausanne, 1015 \\ Lausanne, Switzerland \\ ${ }^{b}$ Laboratory for Neutron Scattering and Imaging, Paul Scherrer Institut, 5232 Villigen, \\ Switzerland \\ ${ }^{c}$ Laboratory for Scientific Developments and Novel Materials, Paul Scherrer Institut, 5232 \\ Villigen, Switzerland \\ ${ }^{d}$ Department of Quantum Matter Physics, University of Geneva, 1211 Geneva, Switzerland \\ ${ }^{e}$ Niels Bohr Institute, University of Copenhagen, 2100 Copenhagen, Denmark
}

\begin{abstract}
A flexible, combined, radial collimator and beryllium (Be) filter have been designed and manufactured at the Paul Scherrer Institut (PSI), Switzerland. The Be is integrated in the radial collimator by placing thin Be slices between the collimator lamellas. The filter/collimator is mounted within a vacuum vessel and dry cooled. The flexible design allows for different degrees of collimation and for different Be lengths. Results of measurements carried out at the BOA beamline at PSI are presented. These experiments include rotation scans determining the focal full width half maximum (FWHM), transmission measurements, test of different collimator lamellas and performance tests of the cooling of the filter. This new combined device will be a crucial part of the CAMEA spectrometer at SINQ, PSI.
\end{abstract}

Keywords: neutrons, radial collimator, Beryllium filter, neutron optics, neutron scattering

\section{Introduction}

2

Inelastic neutron scattering experiments, e.g. at three-axis-spectrometers, require a good signal-to-noise ratio and it is crucial to keep the background as 
4 low as possible to be able to detect even very weak excitations. Thus, collima-

5 tion and filter elements are commonly used in experiments.

6 Most experiments use complex sample environments (SE), e.g for parametric

7 studies under extreme conditions (temperature, high magnetic fields, pressure).

8 Cryo-magnets for example are stabilized by concentric aluminum rings, which

9 generate additional background. Therefore, collimation is required to reduce

10 the background arising from these SE [1]. In the case of instruments with a

11 larger acceptance in scattering angle this would be a radial collimator ( $\mathrm{RC})$.

12 The best performance of a $\mathrm{RC}$ is achieved by placing it as close to the sample

13 as possible. Therefore, the optimal solution would be to include the RC directly

14 into the SE. However, most instruments are designed to work with an existing

15 SE suite, which often does not include such collimation. Thus, the RC should

16 be placed immediately after the SE.

17 Monochromator/analyzer based instruments measuring in the cold neutron regime

${ }_{18}(E=0-20 \mathrm{meV})$, which do not employ a velocity selector, have to take contam-

19 ination by $\lambda / 2$ neutrons into account. Second order scattering by the monochro-

20 mator would not only contaminate the analyzed signal itself, but likewise in-

${ }_{21}$ crease the background [2]. In the case of highly oriented pyrolytic graphite

22 (HOPG) analyzer crystals the analyzers scatter neutrons of higher energies due

23 to parasitic reflections [3-5]. To avoid this effect a filter of polycrystalline, sin-

24 tered Be is employed. Polycrystalline Be scatters neutrons with wave vectors

${ }_{25} k>\frac{\tau}{2}$, with $\tau_{100}=3.19 \AA^{-1}, \tau_{002}=3.51 \AA^{-1}$ and $\tau_{101}=3.64 \AA^{-1}[6,7]$.

26 Smaller neutron wave vectors are transmitted with little attenuation. Thus, to

27 avoid this effect, a Beryllium filter is needed. Since the wavelength transmission

28 of a Be filter above the cut-off is significantly improved at low temperatures,

29 such filters are generally cooled to liquid nitrogen (LN) temperatures $[6,8]$.

30 The suppression of $\lambda / 2$ improves with increasing length of the filter. However,

31 simultaneously the transmission worsens worsens for wavelengths greater than

$324 \AA[9]$. A Be filter length of $120 \mathrm{~mm}$ gives a satisfying compromise between a

з3 $\lambda / 2$ suppression of the order of $10^{-3}$ and overall transmission of roughly $90 \%$.

34 Here, a new implementation of a $\mathrm{RC}$ combined with a Be filter in one compact 
device is presented. Combining both element has two advantages. First, the final device becomes shorter and more compact. Second, the longer collimator blades reduce the poisoning of the filter, i.e. the diffuse leakage of neutrons through the filter, by simply decreasing the open solid angle for neutrons leaking through the filter. Note that there are existing Be filters which employ a coarse collimation, e.g. at RITA-II at SINQ, PSI. However, in conventional filters the coarse collimation is restricted to the dimensions of the Be and allows a simple pressing of the complete stack.

In the design presented here the Be filter is integrated in the front part of the RC (see Fig. 1). Hence, the complete housing of the combined device needs to be cooled to LN temperatures for a good transmission quality. This is especially challenging due to the different thermal expansion coefficients of the various materials used[10, 11]. Most Be filters are conventionally cooled with LN, while the device presented here is dry cooled by a free-piston Stirling cryocooler. Since the performance of such a cooler only slightly depends on its orientation, the cooler can be mounted anywhere around the device. This allows to optimize the position of the cooler for each instrument. Furthermore, the dry cooling opens the possibility to easily construct such collimator/filters with a larger angular coverage. This makes the device especially interesting for the upcoming generation of wide-angle multiplexing spectrometers, e.g. CAMEA [12], BIFROST (formerly CAMEA ESS) [13] and BAMBUS [14], where radial collimation and Be filtering are needed, and spatial restrictions apply. The combined device presented here will be used in the CAMEA spectrometer at PSI.

\section{Description of components}

The design parameters of the filter collimator were chosen in agreement with the requirements specified for the CAMEA spectrometer. Here, dictated by existing SE the front of the collimator has a distance of $550 \mathrm{~mm}$ to the sample position, a length of $280 \mathrm{~mm}$ and a height of $80 \mathrm{~mm}$. A sketch of the assembly is 


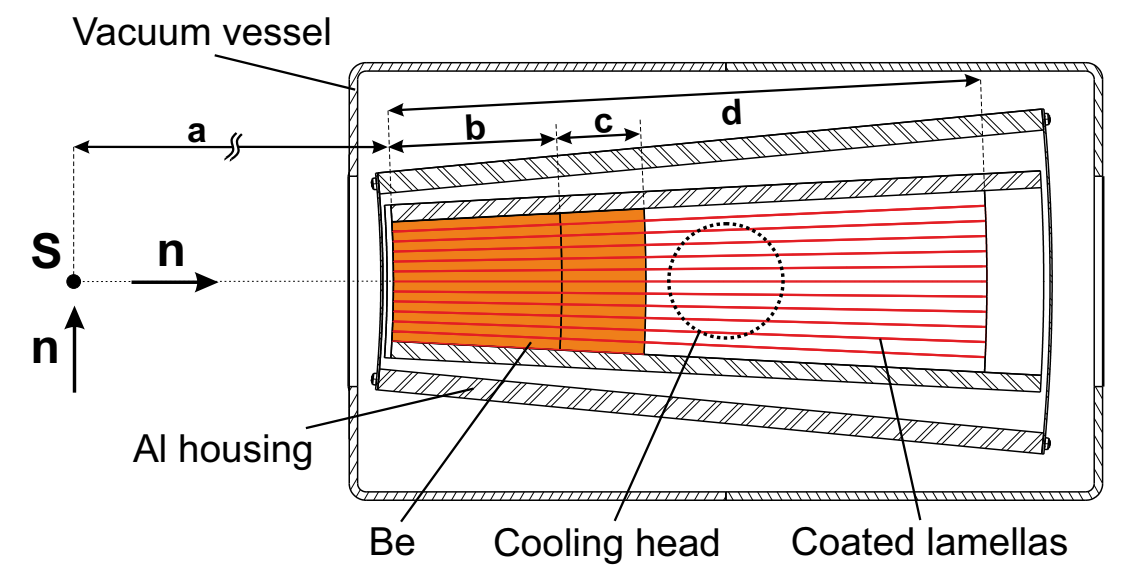

Figure 1: Cut sketch of the combined devise. Neutrons are scattered from the sample (S) towards the collimator. The distance between sample and beginning of the collimator is $a=550 \mathrm{~mm}$ (not to scale). Be wedges (orange, length $b=80 \mathrm{~mm}$ and $c=40 \mathrm{~mm}$ ) can be inserted between the coated lamellas (red, length $d=280 \mathrm{~mm}$ ) to allow for different total Be lengths of $80 \mathrm{~mm}$ and $120 \mathrm{~mm}$. The device is connected to the cooling head (dashed circle), which is mounted on top of the vacuum vessel. shown in Fig. 1. The whole device was mounted within an aluminum housing. 


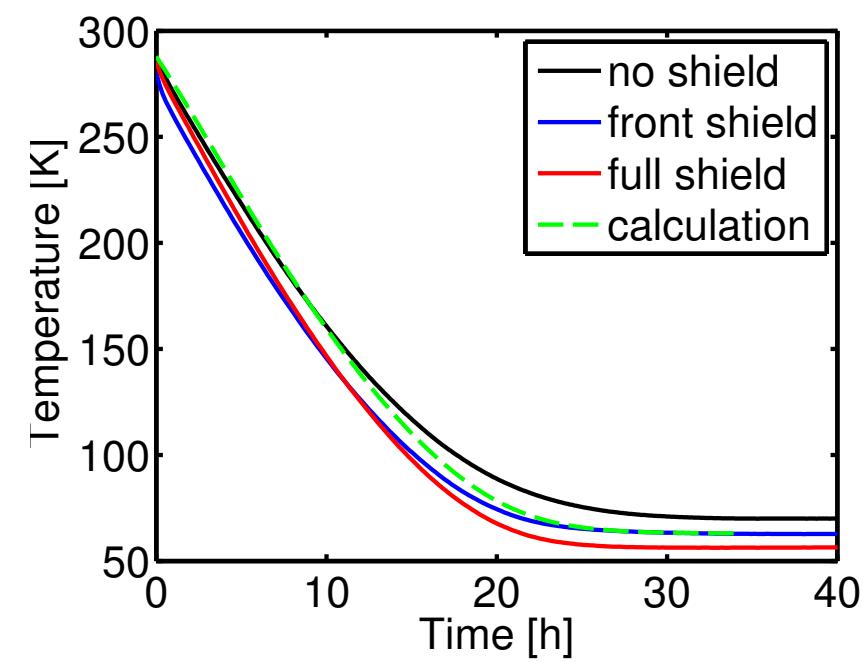

Figure 2: Prototype temperature as a function of cooling time for different heat shield configurations. According to the specifications of the cooling head the heat shield saves a heat load of $2.5 \mathrm{~W}$ on the collimator fins. The calculations (green) were done for a filter with a mass of $12 \mathrm{~kg}$.

81 of the housing. The prototype was mounted within a vacuum vessel and fixed

82 to the cooling head (Cryotel GT, Sunpower, nominal cooling power of $16 \mathrm{~W}$ at

$8377 \mathrm{~K})$.

\section{Experimental results}

85

The characterization experiments were performed at the multi-purpose neu-

${ }_{86}$ tron beamline BOA at SINQ, PSI $[15,16]$. The cooling performance, different

87 collimator configurations and transmission measurements are described in the

88 following sections.

89 3.1. Cooling

90 In order to monitor the cooling process, thermometers were placed at the ${ }_{91}$ housing and directly on the Be. The temperatures as a function of cooling time

${ }_{92}$ is shown in Fig. 2. The initial arrangement (black) had neither heat shields

93 at the front nor at the back of the collimator and the temperature stabilizes

94 after 25 hours around $72 \mathrm{~K}$. In this configuration the collimator fins are exposed 
to heat radiation from the vacuum vessel. Therefore, further cycles were done with radiation shielding (out of thin aluminum foil attached to the frame) only at the front (blue) and shielding at the front, the back and the sides (red). The first configuration stabilizes at a temperature of $63 \mathrm{~K}$ corresponding to a cooling power of $10 \mathrm{~W}$ according to the specifications of the cooling head used. The second configuration stabilized at a temperature of $56 \mathrm{~K}$ corresponding to a cooling power of $7.5 \mathrm{~W}$. Thus, shielding the collimator fins from the heat radiation of the surrounding vessel reduces the heat load by $2.5 \mathrm{~W}$. This seems little, however, in the case of CAMEA the collimator will be 8 times larger resulting in a saving of $20 \mathrm{~W}$ cooling power at LN temperature. Calculations were performed using a lumped heat capacity model. Here, a point mass of $12 \mathrm{~kg}$ of aluminum and the manufacturer's data on the temperature dependent cooling power of the cooler were used. The radiative heat load from the surrounding vessel at RT was considered assuming a surface area of $1800 \mathrm{~cm}^{2}$ with an emissivity of $\epsilon=0.18$. The calculated cooling time (green line in Fig. 2) as well as the base temperature are in excellent agreement with the measurements.

The whole arrangement (equipped with $\mathrm{Si}$ and glass lamellas) was thermally cycled between room temperature (RT) and $60 \mathrm{~K}$ several times in order to check the reliability of the components, mainly the lamellas and the coating. After more than 15 cycles, no damage due to thermal contraction/tension was observed.

\subsection{Transmission Measurements}

The performance of the Be filter was tested using the time-of-flight (ToF) option of BOA. The prototype was mounted in the direct beam and ToF spectra were recorded for Be lengths of $80 \mathrm{~mm}$ and $120 \mathrm{~mm}$, with the filter at RT and cooled to LN temperatures. The spectra were compared to the same open collimator with no Be mounted. The transmission results for the different setups are shown in Fig. 3. As expected, the transmission improves drastically by cooling the Be, e.g. for an incoming wavelength of $\lambda=4.4 \AA$ from $48.2 \%$ to $85.1 \%$ (120 $\mathrm{mm} \mathrm{Be})$ and from $61.6 \%$ to $86.6 \%$ ( $80 \mathrm{~mm} \mathrm{Be})$. At LN tem- 


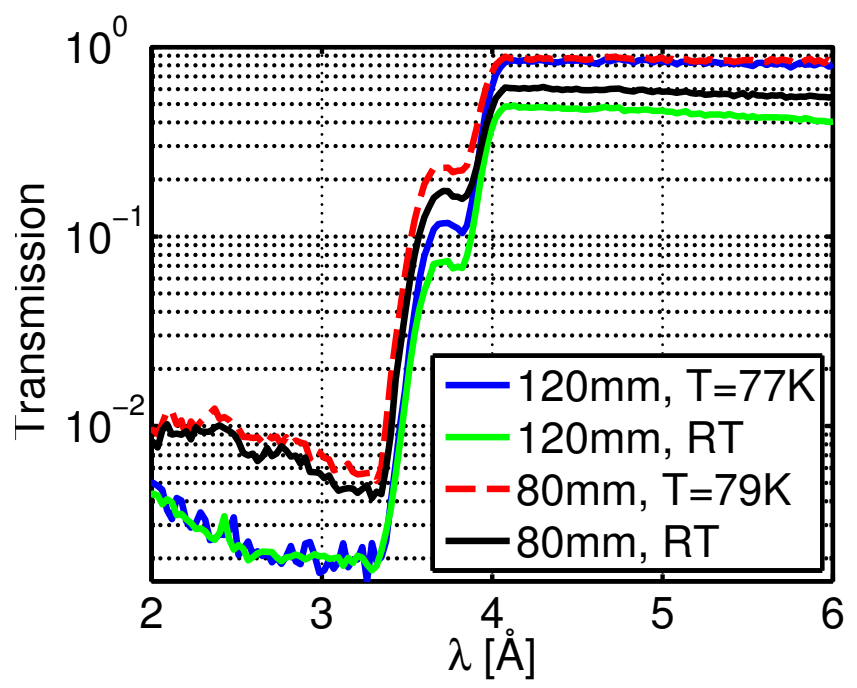

Figure 3: The transmission of the different Be arrangements as a function of incoming wavelength. Cooling the Be (blue, red dashed) shows a significant improvement in transmission compared to Be at room temperature (green, black).

peratures, the normal operation temperature of the filter, the transmission for wavelengths above $4.05 \AA$ is similar for both Be lengths. The experimentally determined absolute transmission above the cut-off is slightly less than the theoretical predictions of $\sim 94 \%(80 \mathrm{~mm})$ and $\sim 92 \%(120 \mathrm{~mm})$ [6], while the ratio is in good agreement. Note that the theoretical calculations do not consider any $\mathrm{BeO}$ content of the filter. Any $\mathrm{BeO}$ content reduces the transmission above the normal Be cut-off due to the shifted cut-off at $\lambda=4.6 \AA$ [17].

There is no sharp cut-off for the suppression of unwanted wavelengths below $4.05 \AA$ but due to several Bragg cut-offs a clear shoulder region is visible. The suppression below the shoulder region is almost temperature independent and it is improved by factors in the range of 3 to 4.5 in the investigated region by increasing the Be length from $80 \mathrm{~mm}$ to $120 \mathrm{~mm}$. A suppression of $\lambda / 2$ neutrons of the order of $10^{-3}$ is achieved. Although cooling the filter improves the transmission the effect is smaller in the shoulder region compared to the region above the cut-off. In the shoulder region an increase in Be length still improves the suppression while above the cut-off almost no effect is visible. However, due to 


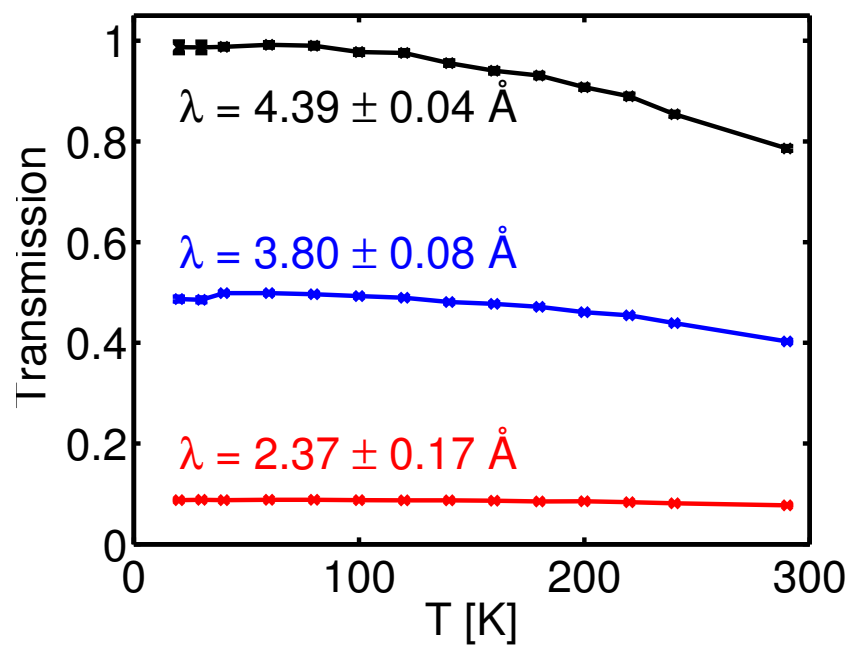

Figure 4: Temperature dependent transmission for wavelengths below and above the Be cut-off $(4.05 \AA)$.

141 the temperature dependent variations in transmission the device should not be

142 operated in the shoulder region.

${ }_{143}$ Additional temperature dependent transmission measurements were performed

144 with a different arrangement using the ToF option of BOA. Here, a smaller

${ }_{145}$ piece of Be (length $40 \mathrm{~mm}$, width $10 \mathrm{~mm}$ ) was mounted in a sample holder and

146 attached to a cryocooler. Spectra were measured for a temperature range of

${ }_{147} T=20-290 \mathrm{~K}$ and normalized to the direct beam spectra. The results for

${ }_{148}$ wavelengths below and above the cut-off and in the shoulder region are shown

${ }_{149}$ in Fig. 4. Here, several time bins were combined to obtain better statistics.

150 For a wavelength below the cut-off $(\lambda=2.37 \pm 0.17 \AA$, red $)$ the transmission

${ }_{151}$ is almost temperature independent. In the shoulder region $(\lambda=3.80 \pm 0.08 \AA$,

152 blue) and above the cut-off $(\lambda=4.39 \pm 0.035 \AA$, black $)$ the relative improvement

153 in transmission is roughly the same $\left(\tau_{\max } / \tau_{\min } \sim 1.2\right)$. Above the cut-off the

154 transmission significantly improves until a temperature of $T=120 \mathrm{~K}$ is reached.

155 Further cooling does not improve the transmission within the statistics. This is

156 far above LN temperatures and demonstrate the advantage of the dry cooling

157 of the filter. Dry cooling allows to optimize the cooling temperature, which 


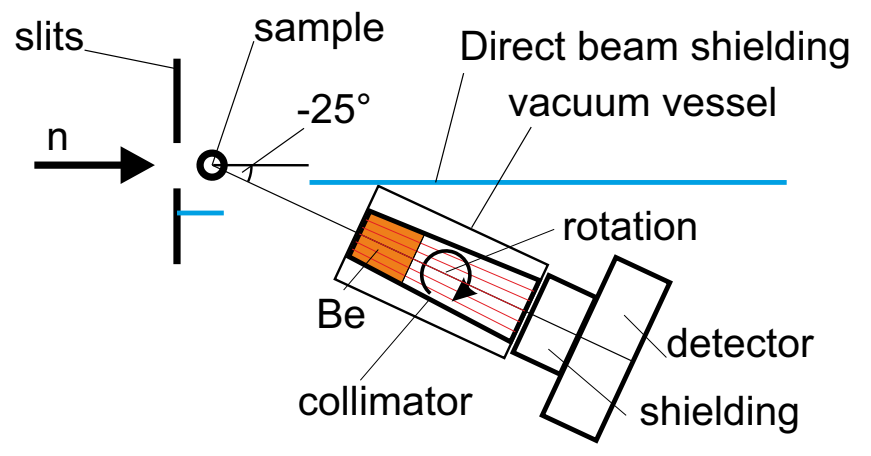

Figure 5: Sketch of the instrument configuration used for the rotation scans. The device (detailed sketch in Fig. 1) is centered to a scattering angle of $-25^{\circ}$ while a Perspex rod was used as an incoherent scatterer. To minimize background from air scattering the direct beam was shielded (blue).

cycles.

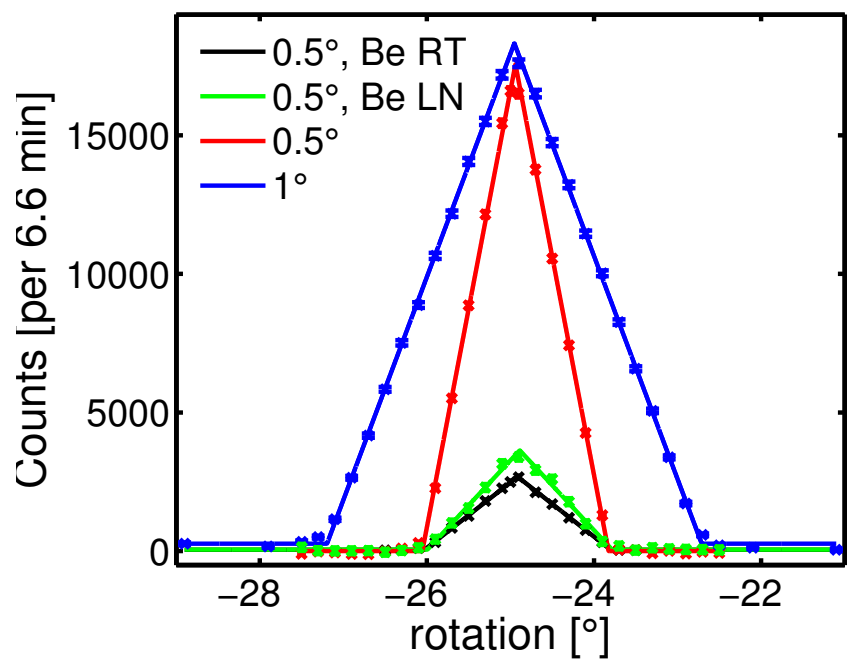

Figure 6: Rotation scans for different collimator configurations using an incoherent scatterer. A triangular function was fitted to the background corrected data. Due to the white beam configuration and changes of the slit opening, the peak intensities cannot directly be compared with each other and are not indicative for the filter performance.

A Perspex rod (diameter $5 \mathrm{~mm}$, length $40 \mathrm{~mm}$ ) was used as an incoherent

scatterer in the white beam. Due to strong background arising from air scat- 


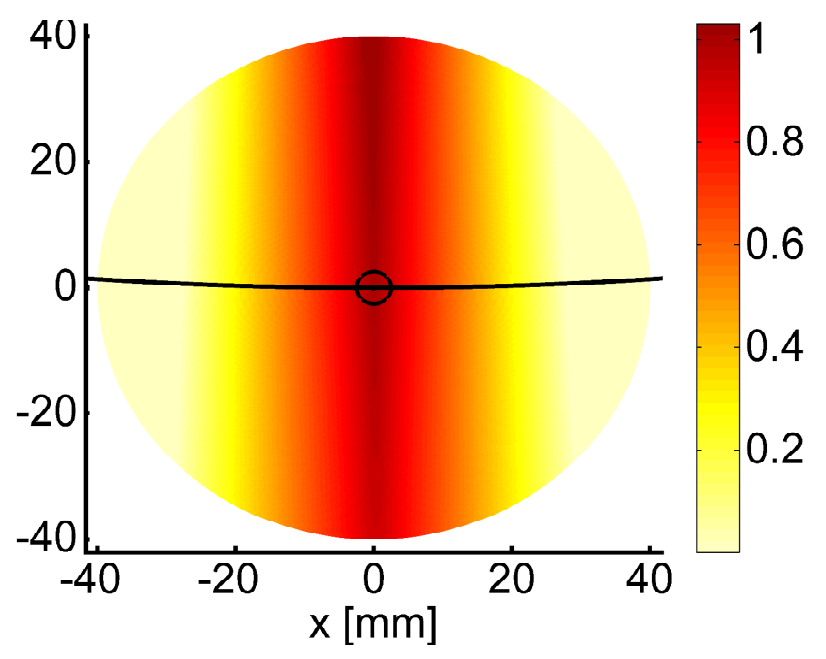

Figure 7: Calculated intensity distribution for a collimation of $1^{\circ}$. The $y$-axis is parallel to the sample-collimator axis. During a rotation scan the sample (black circle) moves along the solid black line.

tering, the direct beam was shielded towards the detector. The collimator con-

figuration was characterized by rotation scans with the collimator centered to a scattering angle of $-25^{\circ}$ (see Fig. 5). The results of the rotation scans are shown in Fig. 6, which displays the results for an empty collimator (collimation of $0.5^{\circ}$ (red) and $1^{\circ}$ (blue)) and $80 \mathrm{~mm}$ Be mounted with $0.5^{\circ}$ collimation at RT (black) and LN temperatures (green). All data were corrected for background and a triangular function was fitted to all data sets. The fitting results are shown in Tab. 1.

The measurements were compared to geometrical calculations of the intensity distribution around the sample region. Here, the opening angle through a single radial collimator channel (focused on the sample position) is calculated for each point around the sample position within the scattering plane. By normalizing 75 the resulting map to the sample position the intensity distribution of an incoherent scatterer through one radial collimator channel is obtained. Neighbored 37 collimator channels are taken into account by adding the same map rotated by 78 the opening angle of the collimator. Fig. 7 shows a calculated example for the 79 $1^{\circ}$ arrangement with a total number of 6 collimator channels. Here, the sample 


\begin{tabular}{|c|c|c|}
\hline Configuration & $\begin{array}{c}\text { FWHM } \\
{[\mathrm{mm}], \text { fit }}\end{array}$ & $\begin{array}{c}\text { FWHM } \\
{[\mathrm{mm}], \text { calc }}\end{array}$ \\
\hline $0.5^{\circ}$ & $13.65(4)$ & 13.81 \\
\hline $1^{\circ}$ & $27.44(5)$ & 27.61 \\
\hline $0.5^{\circ}, 80 \mathrm{~mm} \mathrm{Be}, \mathrm{RT}$ & $13.64(6)$ & 13.81 \\
\hline $0.5^{\circ}, 80 \mathrm{~mm} \mathrm{Be}, \mathrm{LN}$ & $13.48(7)$ & 13.81 \\
\hline
\end{tabular}

Table 1: Fitting results of the different rotation scans using a triangular function. The results are in excellent agreement with the values calculated geometrically.

position is in the center. The y-axis is parallel to the sample-collimator-axis.

The beginning of the collimator is located at $y=550 \mathrm{~mm}$. By performing a rotation scan, the sample position (black circle) w.r.t. the collimator rotation axis is moved along the black solid line. The calculated FWHM values are in excellent agreement with the experimental values (see Tab. 1). The presence of Be within the collimator does not affect the focal width of the collimator. Note that the rotation scans were performed to characterize the collimator performance. Since the measurements were done using a white beam, the peak intensities of these measurements cannot be directly taken as an indicator for the filter performance. In the case of Be inserted into the $\mathrm{RC}$ a large part of the incoming spectrum is cut off by the filter resulting in a larger drop in peak intensity. Furthermore, the slit configuration changed for the Be arrangements at RT and LN temperatures. Thus, these intensities should likewise not be compared directly.

\subsection{Lamellas}

Two lamella materials, Si wafers and boronated glass (Borofloat33, Schott AG), were tested. The lamellas had a thickness of $0.3 \mathrm{~mm}$ and were coated with a $1 \mu \mathrm{m}$ thick, absorbing Gd layer by sputtering. At the center position of the rocking scans (scattering angle of $-25^{\circ}$ ) the collimator without Be was illuminated for $30 \mathrm{~min}$. The results for the intensity of the $1^{\circ}$ configuration integrated over the vertical detector channels are shown in Fig. 8. The intensity structure for the Si lamellas (black) is slightly less pronounced compared to the 


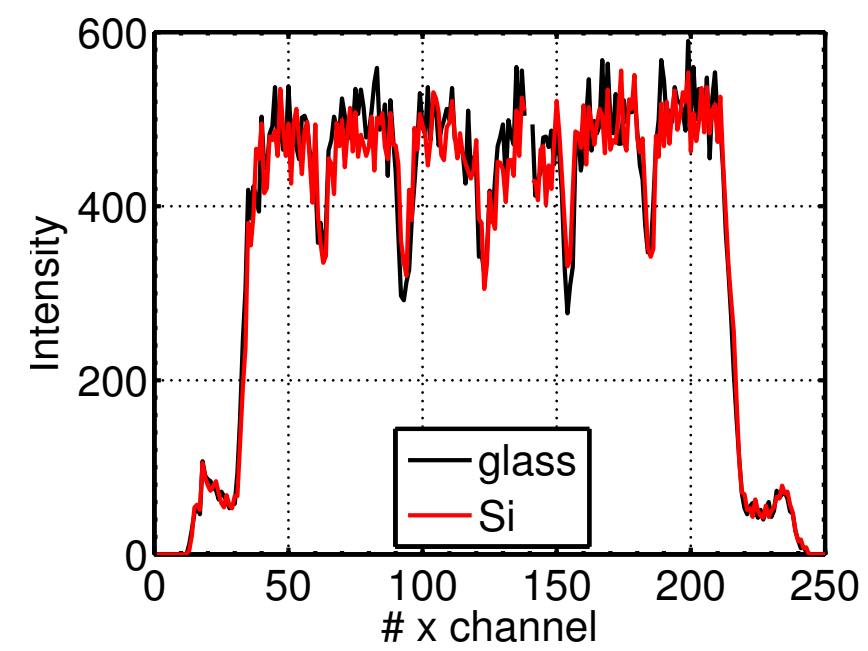

Figure 8: Integrated intensities for different lamella configurations: Si (red) and glass (black). The structure is slightly more pronounced for the glass configuration.

one of the glass lamellas (red). The glass lamellas are less stiff than the Si wafers, which results in a small bending of the lamellas due to their own weight. This increases the effective thickness of the lamellas resulting in a slightly more pronounced intensity structure. The effect is small and in a spectrometer the structure will be smeared out by the mosaicity of the analyzers. Glass lamellas are less expensive compared to Si wafers ( factor of 10$)$. Thus, for the future CAMEA spectrometer glass lamellas coated with ${ }^{10} \mathrm{~B}$ will be used.

\section{Conclusion}

A new, compact, combined device of a radial collimator and a dry cooled Be filter has been successfully tested. It can be shown that such a combined device with the radial collimator much longer than the filter can be realized without sacrificing the performance of the instrument. The achieved collimation is only limited by the manufacturing tolerances of the Be wedges inserted into the radial collimator allowing flexibility in positioning. Furthermore, the dry cooling allows a more flexible operation and installation of the easily extendable layout. This device will be included in the currently constructed CAMEA spectrometer 
at SINQ, PSI. The minimum space requirements are especially interesting for wide angle instruments where spatial restrictions apply, e.g. the upcoming generation of multiplexing neutron spectrometers currently developed at different institutes [12-14].

This project is supported by the Swiss National Science Foundation within R'Equip grant 206021-144972.

[1] T. Krist, Solid state and conventional neutron optical elements, Nuclear Instruments \& Methods in Physics Research Section A - Accelerators Spectrometers Detectors and Associated Equipment 529 (1-3) (2004) 50-53.

[2] R. Toft-Peterson, F. Groitl, D. Quintero-Castro, M. Kure, J. Lim, P. Cernak, S. Alimov, T. Wilpert, M. D. Le, C. Niedermayer, A. Schneidewind, K. Habicht, The first prototype tests of the vertically scattering multiple energy analysis backend for cold triple axis spectrometers, to be published.

[3] E. Frikkee, Application of pyrolytic-graphite as a tunable neutron filter, Nuclear Instruments \& Methods 125 (2) (1975) 307-312.

[4] M. Adib, N. Habib, M. Fathaalla, Neutron transmission through pyrolytic graphite crystals, Annals of Nuclear Energy 33 (7) (2006) 627-632.

[5] M. Adib, N. Habib, I. Bashter, A. Saleh, Neutron transmission through pyrolytic graphite crystal II, Annals of Nuclear Energy 38 (4) (2011) 802807.

[6] N. Habib, Polychristalline beryllium and graphite as cold neutron filters, J. Nuclear \& Radiation Physic. 1 (2) (2006) 137-145.

[7] M. Wahba, On the use of beryllium as thermal neutron filter, Egyptian Journal of Solids 25 (2002) 215-227.

[8] D. C. Tennant, Performance of a cooled sapphire and beryllium assembly for filtering of thermal neutrons, Review of Scientific Instruments 59 (2) (1988) 380-381. 
[9] F. J. Webb, Leakage of thermal neutrons through beryllium filters, Nuclear Instruments \& Methods 69 (2) (1969) 325-\&.

[10] C. A. Swenson, Recommended values for the thermal expansivity of silicon from 0 to 1000 k, Journal of Physical and Chemical Reference Data 12 (2) (1983) 179-182.

[11] K. Wang, R. R. Reeber, The perfect crystal, thermal vacancies and the thermal expansion coefficient of aluminium, Philosophical Magazin A - Physics of Condensed Matter Structure Defects and Mechanical Properties 80 (7) (2000) 1629-1643.

[12] F. Groitl, et al., CAMEA - A novel multiplexing analyzer for neutron spectroscopy, submitted to Review of Scientific Instruments.

[13] P. G. Freeman, J. O. Birk, M. Marko, M. Bertelsen, J. Larsen, N. B. Christensen, K. Lefmann, J. Jacobsen, C. Niedermayer, F. Juranyi, H. M. Ronnow, CAMEA ESS - The continuous angle multi-energy analysis indirect geometry spectrometer for the European Spallation Source, in: QENS/WINS 2014 - 11th International Conference on Quasielastic Neutron Scattering and 6th International Workshop on inelastic neutron spectrometers, Vol. 83 of EPJ Web of Conferences, EDP Sciences, 2015.

[14] J. A. Lim, K. Siemensmeyer, P. Cermak, B. Lake, A. Schneidewind, D. S. Inosov, BAMBUS: a new inelastic multiplexed neutron spectrometer for PANDA, in: M. Zhitomirsky, P. DeReotier (Eds.), International Conference on Strongly Correlated Electron Systems 2014 (SCES2014), Vol. 592 of Journal of Physics Conference Series, IOP Publishing LTD, 2015.

[15] M. Morgano, S. Peetermans, E. Lehmann, T. Panzner, U. Filges, Neutron imaging options at the BOA beamline at Paul Scherrer Institut, Nuclear Instruments and Methods in Physics Research Section A: Accelerators, Spectrometers, Detectors and Associated Equipment 754 (0) (2014) 46 - 56.

[16] http://http://www.psi.ch/sinq/boa/boa. 
[17] M. Adib, N. Habib, I. Bashter, H. Morcos, M. El-Mesiry, M. Mansy, Characteristics of poly- and mono-crystalline $\mathrm{BeO}$ and $\mathrm{SiO} 2$ as thermal and cold neutron filters, Nuclear Instruments and Methods in Physics Research Section B: Beam Interactions with Materials and Atoms 358 (2015) 98 104. 